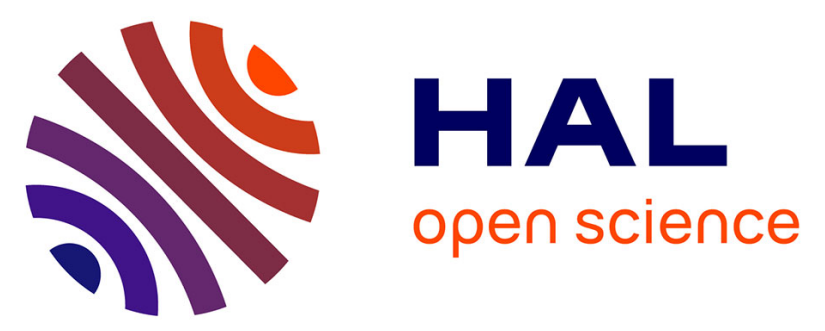

\title{
Generic dynamic modeling and robust Sliding Mode based Model-Free Control for 3D navigation of multirotor VTOL UAVs
}

Yasser Bouzid, Houria Siguerdidjane, Yasmina Bestaoui

\section{- To cite this version:}

Yasser Bouzid, Houria Siguerdidjane, Yasmina Bestaoui. Generic dynamic modeling and robust Sliding Mode based Model-Free Control for 3D navigation of multirotor VTOL UAVs. International Conference on Unmanned Aircraft Systems, ICUAS'18, Jun 2018, Dallas, United States. pp.970-979, 10.1109/ICUAS.2018.8453293 . hal-01811953

\section{HAL Id: hal-01811953 \\ https://hal.science/hal-01811953}

Submitted on 29 Sep 2020

HAL is a multi-disciplinary open access archive for the deposit and dissemination of scientific research documents, whether they are published or not. The documents may come from teaching and research institutions in France or abroad, or from public or private research centers.
L'archive ouverte pluridisciplinaire HAL, est destinée au dépôt et à la diffusion de documents scientifiques de niveau recherche, publiés ou non, émanant des établissements d'enseignement et de recherche français ou étrangers, des laboratoires publics ou privés. 


\title{
Generic dynamic modeling for multirotor VTOL UAVs and robust Sliding Mode based Model-Free Control for 3D navigation
}

\author{
Yasser BOUZID ${ }^{1}$, Houria SIGUERDIDJANE ${ }^{2}$ and Yasmina BESTAOUI ${ }^{1}$
}

\begin{abstract}
This paper introduces a generic and accurate dynamic model, based on Newton-Euler formalism, for multirotor vehicles taking into consideration aerodynamic effects. Besides, the paper considers a reformulation of the well-known ModelFree Control (MFC), which is applied for a low-cost quadrotor even in the presence of various type disturbances, including unmodeled or neglected dynamics, parametric uncertainties, external disturbances, etc. This reformulation takes into consideration the limitation of the estimator used by the classical MFC by using a Sliding Mode auxiliary Controller (SMC) leading to SMC-MFC controller. In addition, instead of using a pure datadriven based control, we introduce the available mathematical dynamics of the system even if they are poorly known. Herein, the MFC principle is employed to deal with the unknown part of the plant only (i.e. unmodeled dynamics, disturbances, etc.). The stability of the closed-loop system is guaranteed and for which a theoretical analysis is provided. The numerical simulations have shown satisfactory results. An in-depth discussion, with respect to the control performance and consumed energy, is highlighted by considering several scenarios and using several metrics.
\end{abstract}

\section{INTRODUCTION}

\section{A. Short review}

The Unmanned Aerial Vehicles (UAVs) are traditionally used in the military for diverse classical missions such as real-time video reconnaissance, inspection, surveillance, etc. Recently, they are being increasingly used in civilian domains in various applications such as environmental and weather monitoring, fire detection, pollutant estimation and coverage [1].

The involving of UAVs, in the ease and profit of the human life, has attracted the attention of industries and academia around the world to deal with the new challenges, which leads to immense advances and fast-growing in the field of autonomous flying robots. Indeed, a broad range of prototypes have been constructed, in the last two decades, motivated by the technological progress in electrical motors, embedded electronics, wireless communication, etc. Several monographs such as [2] may give the readers some basic understanding of each UAV type and the related issues.

Among the broad range of UAVs platforms, the Vertical Take-Off and Landing (VTOL) multirotors have a special form that involves pairs of counter-rotating rotors to provide lift and directional control. Due to the structural properties

\footnotetext{
${ }^{1}$ Y. Bouzid and Y. Bestaoui are with IBISC, Université d'Evry Val d'Essonne, Université Paris-Saclay, Evry, 91000, France yasserempegmail.com, Yasmina.Bestaouisebbane@univ-evry. fr

${ }^{2} \mathrm{H}$. Siguerdidjane is with $\mathrm{s}$ with L2S, CentraleSupélec, Université Paris-Saclay, Gif sur yvette, 91190, France Houria.Siguerdidjanedcentralesupelec.fr
}

of the multirotor dynamics: under-actuated, highly coupled and highly nonlinear and hierarchical system, a huge amount of advanced control strategies, such as nonlinear internal model control [3], energy based control [4], Immersion and Invariance [5], etc. have been proposed as flight control systems. In fact, flight control field is well developed and can offer many solutions. There is a rich literature describing different control techniques (see as for instance [6]).

\section{B. Motivations \& contribution}

Notice that the multirotor vehicles are very sensitive to the disturbances especially the wind, during outdoor manipulations. Most of the implemented control laws are modelbased control strategies that require a complete knowledge of the system and its surrounded phenomena. Therefore, these control techniques, initially designed in the ideal case, considering an accurate model, behave with less efficiency in the presence of some disturbances then leading to poor performance even if the controller exhibits robustness features.

Our poor knowledge of the disturbed model may produce poor control performance and lead to the instability of the system in more extreme conditions making the control tasks more challenging. To alleviate the above issues, we distinguish, in the literature, more sophisticated tools that have been proposed and can be classified into three main categories based on distinct principles: 1) adaptation principle such as the controller proposed in [7] for steering a quadrotor vehicle along a trajectory, while rejecting constant force disturbances, 2) on-line estimation \& observation as the Active Disturbance Rejection Control (ADRC) algorithm detailed in [8] by means of extended state observer (ESO) and 3) sliding mode framework as for instance the controller developed in [9], which is combined with a backstepping technique for the sake of trajectory tracking.

Recently, a Model-Free Control (MFC) technique based on a continuous updating of the input-output behavior of a very-local model is proposed. The main advantage of the MFC strategy is that it does not require knowledge of the system dynamics. Its anticipation property makes the control possible even with the presence of disturbances [10]. It is employed recently, in many real cases such as planar manipulator [11], mobile robot [12] and quadrotors [13].

To avoid the difficulties in obtaining an accurate multirotor model, a revisited formulation of the classic MFC is used in this paper to control the multirotor vehicles. The first step is to consider a mathematical model of the system affected by some uncertainties and disturbances. After a compensation 
of the known dynamics, an MFC principle is applied to deal with the unknown part of the system to simultaneously accommodate the unmodeled (neglected) dynamics and the disturbances. However, the steady-state error, in this case, depends on the accuracy of the unknown dynamics estimation. In order to achieve a robust and accurate tracking error, a sliding mode based control is added to MFC, thus obtaining an efficient and robust controller of multirotors.

The development of a matched model is highly important to validate and test the effectiveness of the various control laws. Rich literature shows considerable advances in the subject of modeling such as Altug et al. [14] and Wang [15]. Thus, in this paper, a complete and generic mathematical modeling of the dynamic behavior of multirotors is proposed, which is valid whatever the multirotors typology. Our modeling is a rectified version of Wang's model [15] that is proposed for quadrotors. Moreover, Wang includes the hub forces without paying attention to the fact that these forces generate a moment about $Z$-axis. This moment has been taken into account in our model. Moreover, the flapping moment and its effect are included in this paper. To validate the designed controllers, a simplified model is first used prior applying them on the complete model.

\section{Outlines}

The remainder of this paper is organized as follows: in Section II, the multirotors structure is discussed. Section III details a generic dynamic model of multirotor vehicles. Section IV introduces the design of our nonlinear control approach. The simulation results are illustrated in Section V. Finally, concluding remarks are given.

\section{StRUCtURAL OPERATIONS}

The multirotor vehicles have almost the same principle of flight. They are differentiated by the placement and the size of arms as well as the number of rotors. Indeed, all of them can be designed, doing some structural operations that we classify below, from the main configuration of a quadrotor (see Figure 1).

The multirotors considered in this paper are those with an even number of rotors $\left(2 N_{r} \geq 4\right.$ where $N_{r} \in \mathbb{N}$ is the number of pairs). For the sake of symmetry, each pair of rotors are placed on two opposite sides of the airframe. We stress also that the axis of rotation of the rotors are parallel to $Z_{B}$-axis (see Figure 1).

The quadrotor in Figure 1 is the most widely used configuration. It is controlled to fly in "X-flight configuration", i.e. pointing two arms in the longitudinal navigation direction, which is better suited for aerial photography. It has two pairs of rotors (propellers) $\left(N_{r}=2\right)$ where two rotors spin in the clockwise direction $\left(S_{p}=1\right)$ whilst the other two spin anticlockwise direction $\left(S_{p}=-1\right)$. Each rotor is mounted on a separated arm of length $\left.l_{i}\right|_{i=1, \ldots, 2 N_{r}}=l_{M}$. The angles between the successive arms, $\left.\alpha_{i, i+1}\right|_{i=1, \ldots, N_{r}-1}$, are considered as characteristic angles that equals for this main configuration to $\left.\alpha_{i, i+1}\right|_{i=1, . ., N_{r}-1}=\alpha_{M}$. Changing the speed of the four rotors, the quadrotor will produce different motions (roll, pitch, yaw, altitude) with six degrees of freedom. The throttle input is the sum of the thrusts generated by each rotor. This main configuration leads to many other possible configurations.

1) Rotation around the $Z_{B}$-axis: By rotating the main configuration of the quadrotor in Figure 1 around the $Z_{B^{-}}$ axis by an angle $\alpha_{M} / 2=\pi / 4$, we get another famous configuration called +shaped quadrotor i.e. one arm is oriented into the longitudinal navigation direction (see Figure 2a).

2) Variation of the characteristic angles: By varying the characteristic angles $\left.\alpha_{i, i+1}\right|_{i=1, \ldots, N_{r}-1}$, we get new configurations. For the quadrotor main configuration, we obtain just one form called $\mathrm{H} 4$ - shaped quadrotor, which is displayed in Figure 2b. The $H 4-$ shaped quadrotor provides some benefits for specific applications such as aerial photography and allows more space to place equipment and battery.

3) Variation of the arms length: By changing the length of arms $\left.l_{i}\right|_{i=1, \ldots, 2 N_{r}}$, we get new configurations. We stress that for the sake of symmetry, the lengths are not changed arbitrarily. An example is shown in Figure 2c. This configuration is called $V 4-$ shaped quadrotor, which is an unconventional quadrotor design. This configuration is proposed to reduce the airframe drag or if more responsive yaw control is required. Moreover, it allows wider field of view. The unsymmetrical design will make it slightly harder to balance the Center of Gravity (CoG) properly.

4) Coaxial rotors: At the same axis of rotation for each rotor, we can add another rotor that rotates inversely. For the quadrotor main configuration, we obtain an octorotor called $X 8$ coaxial octorotor as presented in Figure 2 d. It has higher thrust to weight ratio among the most common ones. Using this redundant quadrotors, the chances of a safe return in case of actuator failure is also significantly increased but will result in a significant decrease in attitude control performance.

5) Variation of the rotors number: If we increase the number of the rotors pairs $N_{r}$, starting from the quadrotor main form, we get a main configuration of an hexarotor, which is shown in Figure 2e. Hexacopters are generally used when quadrotors aren't strong enough or when some limited redundancy is required because of expensive equipment. This redundancy allows a safe landing in the case of emergences. The overall mass and inertia of the multirotor will increase because of the additional arms and rotors, which will increase the stability of the system as well as its payload capacity. However, the agility of the aircraft will be limited. This configuration becomes among the popular platforms. If the same operation is applied to the hexarotor, an ocotrotor with main configuration is obtained (see Figure $2 \mathrm{~h}$ ). This configuration becomes also a popular one.

Applying these operations to the resulting configurations, new shapes are obtained where some of them is shown in Figure 2. 


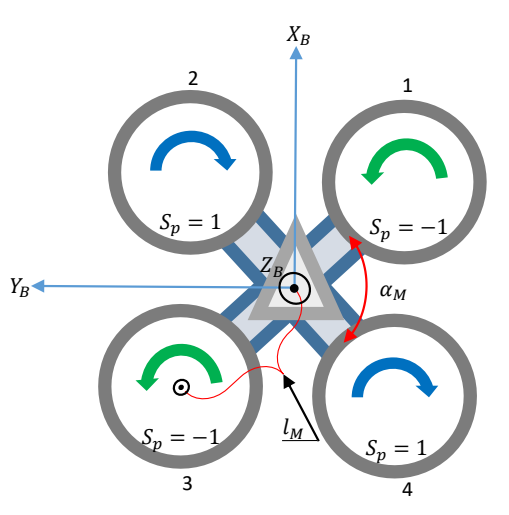

Fig. 1: Quadrotor main configuration.
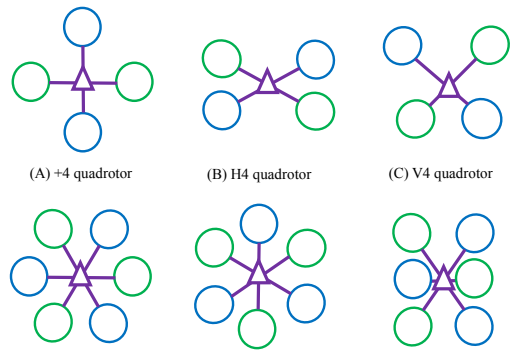

(C) V4 quadrotor

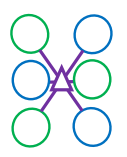

(G) H6 haxrotor
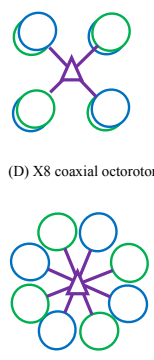

(H) X8 ocotorotor

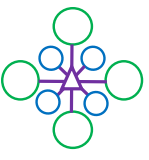

(I) 88 Ocotorotor

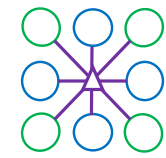

(J) $\square 8$ ocotoroto

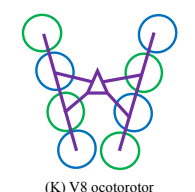

(K) V8 ocotorotor

Fig. 2: Structural applied operations.

\section{MATHEMATICAL MODELING AND EQUATIONS OF MOTION}

The vehicle operates in two coordinate frames as shown in Figure 3: the Earth-fixed frame $R_{E}\left(O_{E}, X_{E}, Y_{E}, Z_{E}\right)$ and the Body-fixed frame $R_{B}\left(O_{B}, X_{B}, Y_{B}, Z_{B}\right) . R_{E}$ is regarded as an inertial reference frame in which Newton's laws of motion are valid and $R_{B}$ is attached to the vehicle and constrained to move with it where the vehicle is considered as rigid body based on the following assumptions [15]:

Assumption 1: The structure and the propellers are rigid and perfectly symmetrical.

Assumption 2: The dynamic of the rotors is relatively fast and therefore it will be neglected.

The origin of the Body-fixed frame $O_{B}$ is an invariant point and belongs to the vehicle structure. To simplify, it is chosen to be the vehicle Center of Gravity (CoG). The attitude of the multirotor aerial vehicle is described in the Earth-fixed frame adopting the Tait-Bryan angles.

Starting from the Body-fixed frame to the Earth-fixed frame, the sequence of rotations chosen is: the $X-Y-Z$ sequence, meaning that the attitude is obtained first by the

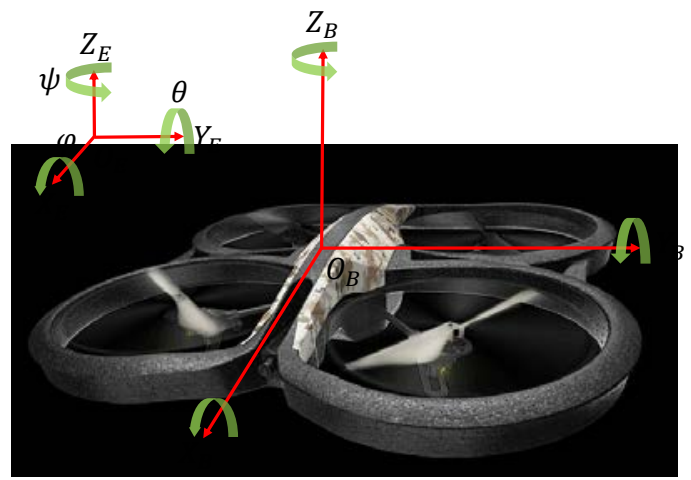

Fig. 3: Frames and rotations representation.

roll angle " $\varphi$ ", then by the pitch angle " $\theta$ " and then by the yaw " $\psi$ ". The obtained rotation matrix $\mathcal{R}$ is given by

$$
\mathcal{R}(\varphi, \theta, \psi)=\left[\begin{array}{cccc}
\mathrm{c}_{\psi} \mathrm{c}_{\theta} & \mathrm{c}_{\psi} \mathrm{s}_{\theta} \mathrm{s}_{\varphi} & \mathrm{s}_{\psi} \mathrm{c}_{\varphi} & \mathrm{c}_{\psi} \mathrm{s}_{\theta} \mathrm{c}_{\varphi}+\mathrm{s}_{\psi} \mathrm{s}_{\varphi} \\
\mathrm{s}_{\psi} \mathrm{c}_{\theta} & \mathrm{s}_{\psi} \mathrm{s}_{\theta} \mathrm{s}_{\varphi}+\mathrm{c}_{\psi} \mathrm{c}_{\varphi} & \mathrm{s}_{\psi} \mathrm{s}_{\theta} \mathrm{c}_{\varphi} & \mathrm{c}_{\psi} \mathrm{s}_{\varphi} \\
\mathrm{s}_{\theta} & \mathrm{c}_{\theta} \mathrm{s}_{\varphi} & \mathrm{c}_{\theta} \mathrm{c}_{\varphi}
\end{array}\right]
$$

where $\mathcal{R} \in S O(3)=\left\{\mathcal{R} \in \mathbb{R}^{3 \times 3} \mid \mathcal{R}^{T} \mathcal{R}=I_{3 \times 3}, \operatorname{det}(\mathcal{R})=\right.$ $1\}$. The subscript $3 \times 3$ means the dimension of the matrix is $3 \times 3$ and $I_{3 \times 3}$ is the identity matrix of dimension $3 . s_{(.)}$and $c_{(.)}$are abbreviations for $\sin ($.$) and \cos ($.$) respectively.$

Based on the previous description, the transformation between the world frame and the body frame can be expressed explicitly using the rotation matrix $\mathcal{R}$. So, the velocity vector $V^{B}=(u, v, w)^{T}$ of the vehicle expressed in a Body-fixed frame can be rotated into the Earth-fixed frame as follows

$$
\dot{\chi}=\mathcal{R}(\varphi, \theta, \psi) V^{B}
$$

where $\dot{\chi}=(\dot{x}, \dot{y}, \dot{z})^{T}$ is the velocity vector of the multirotor in the inertial frame $R_{E}$.

The relation between the angular velocities $\varpi=(p, q, r)^{T}$ of the multi-rotor in the Body-fixed frame and the angular velocities $\dot{\eta}=(\dot{\varphi}, \dot{\theta}, \dot{\psi})^{T}$ in the Earth-fixed frame is presented as [16]:

$$
\dot{\eta}=\left[\begin{array}{ccc}
1 & \mathrm{~s}_{\varphi} \tan \theta & \mathrm{c}_{\varphi} \tan \theta \\
0 & \mathrm{c}_{\varphi} & -\mathrm{s}_{\varphi} \\
0 & \mathrm{~s}_{\varphi} \sec \theta & \mathrm{c}_{\varphi} \sec \theta
\end{array}\right] \varpi
$$

Obviously, Tait-Bryan angles representation suffers from some singularities: $\varphi= \pm \frac{\pi}{2}, \theta= \pm \frac{\pi}{2}$. In practice, this limitation does not affect the multirotor in normal flight mode.

\section{A. Rigid body dynamics}

The rigid body has six degrees of freedom, with a mass $m$ and an inertia $I \in \mathbb{R}^{3 \times 3}$ about the center of gravity. Let $V^{B}=(u, v, w)^{T} \in \mathbb{R}^{3}$ denotes the linear velocity of the center of gravity and $\varpi=(p, q, r) \in \mathbb{R}^{3}$ its angular velocity expressed in the Body-fixed frame. Let $\eta=(\varphi, \theta, \psi)^{T} \in \mathbb{R}^{3}$ describes the orientation of the rigid body (Roll, Pitch, Yaw) and $\chi=(x, y, z)^{T} \in \mathbb{R}^{3}$ denotes its absolute position with respect to $R_{E}$. Notice that $\chi$ and $\eta$ can be regarded as translational and rotational coordinates 
respectively. Therefore, the relation between the velocities and the external forces $\mathcal{F}^{B}=\left(\mathcal{F}_{x}^{B}, \mathcal{F}_{y}^{B}, \mathcal{F}_{z}^{B}\right)^{T} \in \mathbb{R}^{3}$ and moments $\mathcal{M}^{B}=\left(\mathcal{M}_{x}^{B}, \mathcal{M}_{y}^{B}, \mathcal{M}_{z}^{B}\right)^{T} \in \mathbb{R}^{3}$, applied to CoG, expressed in $R_{B}$ frame, is written using Newton-Euler formalism as

$$
\left[\begin{array}{cc}
m I_{3 \times 3} & O_{3 \times 3} \\
O_{3 \times 3} & I
\end{array}\right]\left[\begin{array}{c}
\dot{V}^{B} \\
\dot{\varpi}
\end{array}\right]+\left[\begin{array}{c}
\varpi \times m V^{B} \\
\varpi \times I \varpi
\end{array}\right]=\left[\begin{array}{c}
\mathcal{F}^{B} \\
\mathcal{M}^{B}
\end{array}\right]
$$

The symbol $O_{3 \times 3}$ means a $3 \times 3$ dimensional zero matrix and $\times$ denotes the cross product. Based on Assumption 1 and with an appropriate choice of the Body-fixed frame as represented in Figure 3, the inertia matrix is diagonal $I=$ $\operatorname{diag}\left(I_{x}, I_{y}, I_{z}\right)$. The next step is to determine the forces $\mathcal{F}^{B}$ and the moments $\mathcal{M}^{B}$.

\section{B. Description of the multirotor vehicles}

In the previous subsection, we have deduced the NewtonEuler equations for a rigid body system, which are suitable to describe any multirotor system. The main difference, essentially, lies in various aerodynamic forces and moments that are defined in function of the rotors speeds through the shape of the vehicle and its number of rotors. Before presenting the main external forces and moments, we provide first a global description of the multirotor vehicles considered in this paper.

The multirotor vehicles have almost the same principle of flight. They are differentiated by the placement and the size of arms as well as the number of rotors. Let $\Re_{O}=$ $\left\{r o_{i}, i=1, \ldots, 2 N_{r}\right\}$ be the set of rotors where $N_{r} \in \mathbb{N}$ is the number of pairs of rotors (propellers) with $2 N_{r} \geq 4$ i.e. the considered multirotors are those with even number of rotors. Each rotor $\left.r o_{i}\right|_{i=1, \ldots, 2 N_{r}}$ located at $\left.o_{i}\right|_{i=1, \ldots, 2 N_{r}}$ at the same plane as the $\mathrm{CoG}$, is supported by an arm of length $\left.l_{i}\right|_{i=1, \ldots, 2 N_{r}}$ and rotates around an axis, which is parallel to the axis $Z_{B}$ (the rotors are not tilted). Thus, a rotor $r o_{i}$ is defined by polar coordinates $o_{i}\left(l_{i}, \alpha_{i}\right) \in \mathbb{R} \times[0,2 \pi]$ where $\alpha_{i}$ denotes the angle between its arm and the axis $X_{B}$, rotation speed $\Omega_{i}$ and a sense of spinning where $N_{r}$ rotors spin in the clockwise direction $\left(S_{p}=1\right)$ whilst the others spin in the anticlockwise direction $\left(S_{p}=-1\right.$ ) (see in Figure 4 the representation of one rotor). For the sake of symmetry, each pair of rotors are placed in two opposite sides of the airframe $\left(\alpha_{i+N_{r}}=\alpha_{i}+\pi, i=1, \ldots, N_{r}\right)$ with the same arms length $\left(l_{i}=l_{N_{r}+i}, i=1, \ldots, N_{r}\right)$. This description is valid for a large class of multirotor vehicles that allows us, later, to provide a global expression of the aerodynamic forces and moments in function of the rotors speeds.

\section{Applied Forces and moments to the multirotor}

1) Forces: Neglecting the ground effects, we list three main forces acting on the mulirotor. The first one is the gravity force $G=-m g$. It is along the axis $Z_{E}$ in the negative direction where $g$ is the gravity coefficient. Besides, each rotor ro $_{i}$ produces thrust \& hub forces, which depend on its angular velocity $\Omega_{i}$. The thrust force $T_{i}$ is along the $Z_{B}$-axis in the positive direction whilst the hub force $H_{i}$ is in the plane $X_{B} Y_{B}$ on the negative direction of the

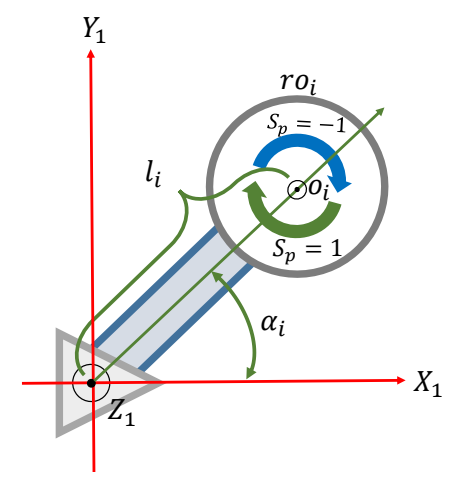

Fig. 4: Rotor description.

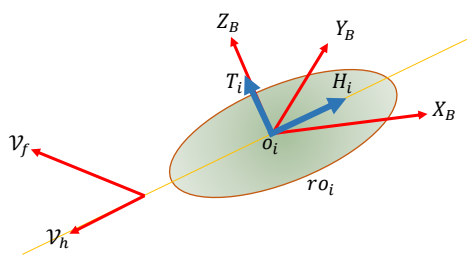

Fig. 5: Forces induced by rotor.

horizontal velocity $\mathcal{V}_{h}$ (the projection of the forwarding velocity $\mathcal{V}_{f}$ in the $X_{B} Y_{B}$ plane). Thus, the hub force can be decomposed into two components $H_{x i}$ along $X_{B}$-axis and $H_{y i}$ along $Y_{B}$-axis in the Body-fixed frame. Graphical representation of these forces is displayed for one rotor in Figure 5. Obviously, the total thrust and hub are the sum of forces generated by each rotor. As the multirotor has $2 N_{r}$ rotors, forces on a multirotor are:

$$
T=\sum_{i=1}^{2 N_{r}} T_{i}, H_{x}=-\sum_{i=1}^{2 N_{r}} H_{x i}, H_{y}=-\sum_{i=1}^{2 N_{r}} H_{y i}
$$

Once the main forces have been reviewed, the total external force vector acting on the vehicle is expressed in the Body-fixed frame, using the rotation matrix (1) as:

$$
\begin{aligned}
\mathcal{F}^{B} & =\left[\begin{array}{c}
H_{x} \\
H_{y} \\
T
\end{array}\right]+\mathcal{R}^{T}\left[\begin{array}{l}
0 \\
0 \\
G
\end{array}\right] \\
& =\left[\begin{array}{c}
-\sum_{i=1}^{2 N_{r}} H_{x i} \\
-\sum_{i=1}^{2 N_{r}} H_{y i} \\
\sum_{i=1}^{2 \bar{N}_{r}} T_{i}
\end{array}\right]+\mathcal{R}^{T}\left[\begin{array}{c}
0 \\
0 \\
-m g
\end{array}\right]
\end{aligned}
$$

where $\mathcal{R}^{-1}=\mathcal{R}^{T}$.

2) Moments: Herein, we present the main external moments that are expressed in the Body-fixed frame and their directions. Roll and pitch moments, $\mathcal{M}_{\varphi}$ and $\mathcal{M}_{\theta}$, around $X_{B}$ and $Y_{B}$-axes respectively, are achieved by the difference in combined thrusts in the opposite sides of the vehicle. The moments about the shaf, $Q$, makes the multirotor turns about 
the $Z_{B}$-axis. There are $2 N_{r}$ rotors that are separated with different rotational directions (clockwise and anti-clockwise directions) inducing moments in the different direction. The direction of the moments is decided according to the right hand rule. The total moments are given by:

$$
\mathcal{M}_{\varphi}=\sum_{i=1}^{2 N_{r}} l_{i} \mathrm{~s}_{\alpha_{i}} T_{i}, \mathcal{M}_{\theta}=-\sum_{i=1}^{2 N_{r}} l_{i} \mathrm{c}_{\alpha_{i}} T_{i}, Q=\sum_{i=1}^{2 N_{r}} S_{p i} Q_{i}
$$

where $S_{p i}=1$ if ro $_{i}$ rotates in the clockwise direction and $S_{p i}=-1$ if ro $_{i}$ rotates in the anti-clockwise direction.

As presented previously, the hub forces have two components along the $X_{B}$-axis and $Y_{B}$-axis. These hub forces exercise moments on the vehicle around the $Z_{B}$-axis. These two components contribute on the moment according to their locations $\left.o_{i}\right|_{i=1, \ldots, 2 N_{r}}$. Therefore, the total hub moment is given by

$$
\mathbb{H}=\sum_{i=1}^{2 N_{r}} l_{i} \mathrm{~s}_{\alpha_{i}} H_{x i}-\sum_{i=1}^{2 N_{r}} l_{i} \mathrm{c}_{\alpha_{i}} H_{y i}
$$

Rotations around two axes bring into a third rotation around an axis, which is perpendicular to the plane formed by the two former axes. This effect is called the gyroscopic effect. Therefore, when a multirotor rotates along the axis $X_{B}$ in the body frame, a moment along the $Y_{B}$-axis is created on each rotating rotor. When a multirotor rotates along $Y_{B}$-axis in the body frame, each rotor also has a moment along $X_{B}$. The direction of moments is set according to right-hand rule. The total gyroscopic components are:

$$
\mathcal{G}_{x}=J_{r} q \sum_{i=1}^{2 N_{r}} S_{p i} \Omega_{i}, \mathcal{G}_{y}=-J_{r} p \sum_{i=1}^{2 N_{r}} S_{p i} \Omega_{i}
$$

where $J_{r}$ is the inertia of a rotor.

During the translational flight, there is a difference in blade lift between the advancing and retreating blades. This effect is called the blade flapping. The difference of the lifts applies a moment to the rotor disk. The flapping moment $\mathcal{B}_{i}$ is perpendicular to the rotor shaft and the forward velocity of the multirotor. The flapping moments of each rotor $\mathcal{B}_{i}$ can be separated into moments $\mathcal{B}_{x i}$ and $\mathcal{B}_{y i}$. The total blade flapping moment of a multirotor is:

$$
\mathcal{B}_{x}=\sum_{i=1}^{2 N_{r}} S_{p i} \mathcal{B}_{x i}, \mathcal{B}_{y}=\sum_{i=1}^{2 N_{r}} S_{p i} \mathcal{B}_{y i}
$$

Once, the various moments affecting the multirotor are presented, the total moment vector is presented in the Bodyfixed frame as:

$$
\begin{aligned}
& \mathcal{M}^{B}=\left[\begin{array}{c}
\mathcal{M}_{\varphi}+\mathcal{G}_{x}+\mathcal{B}_{x} \\
\mathcal{M}_{\theta}+\mathcal{G}_{y}+\mathcal{B}_{y} \\
Q+\mathbb{H}
\end{array}\right] \\
& =\left[\begin{array}{c}
\sum_{i=1}^{2 N_{r}} l_{i} \mathrm{~s}_{\alpha_{i}} T_{i}+J_{r} q \sum_{i=1}^{2 N_{r}} S_{p i} \Omega_{i}+\sum_{i=1}^{2 N_{r}} S_{p i} \mathcal{B}_{x i} \\
-\sum_{i=1}^{2} N_{r} l_{i} \mathrm{c}_{\alpha_{i}} T_{i}-J_{r} p \sum_{i=1}^{2} N_{r} S_{p i} \Omega_{i}+\sum_{i=1}^{2} N_{r} S_{p i} \mathcal{B}_{x i} \\
\sum_{i=1}^{2 N_{r}} S_{p i} Q_{i}+\sum_{i=1}^{2 N_{r}} l_{i} \mathrm{~s}_{\alpha_{i}} H_{x i}-\sum_{i=1}^{2 N_{r}} l_{i} \mathrm{c}_{\alpha_{i}} H_{y i}
\end{array}\right]
\end{aligned}
$$

Usually, the control problems are studies considering the motion of the vehicle around its $\mathrm{CoG}$ and the relative motion of the CoG with respect to the inertial frame $R_{E}$. Therefore, the translational velocities and accelerations are defined in the Earth-fixed frame $R_{E}$ while the angular velocities and accelerations are defined in the Body-fixed frame $R_{B}$. This simplifies the dynamics equations.

The different forces and moments, presented in the previous paragraphs, are expressed in the Body-fixed frame. Therefore, just the forces are rotated into the Earth-fixed frame using rotation matrix (1).

\section{Control oriented model}

Usually, for many scenarios and applications, hovering over a point or flying with moderate speeds are considered where the tilt angles are not excessive. There is no need of aggressive maneuvers or violent displacement. In this context, we are allowed to approximate the Euler angular velocities by the vehicle angular velocities:

$$
\dot{\varphi} \approx p, \quad \dot{\theta} \approx q, \quad \dot{\psi} \approx r
$$

Physically, the multirotor vehicle is controlled through the rotational speeds $\left.\Omega_{i}\right|_{i=1, \ldots, 2 N_{r}}$ of the $2 N_{r}$ rotors where the thrust forces and the yawing moments are the main elements of control. As claimed by Bangura \& Mahony [17], in conditions of flight as stated above, the aerodynamic coefficients can be considered as constants, which are identified from static tests. Therefore, it is often considered that the main thrust and yawing moment are proportional to the square of the propeller's speeds (see as for instance [18]):

$$
T_{i}=b \Omega_{i}^{2}, \quad Q_{i}=d \Omega_{i}^{2}
$$

where $b$ and $d$ are static aerodynamic coefficients.

As known, the multirotor vehicles have four control inputs $u=\left(u_{z}, u_{\varphi}, u_{\theta}, u_{\psi}\right)^{T}$ that are the total thrust, the rolling moment, the pitching moment and the yawing moment:

$$
u_{z}=T, \quad u_{\varphi}=\mathcal{M}_{\varphi}, \quad u_{\theta}=\mathcal{M}_{\theta}, \quad u_{\psi}=Q
$$

Considering the new formulations of $T_{i}$ and $Q_{i}$ given by (12), the control inputs become

$$
\begin{array}{r}
u_{z}=b \sum_{i=1}^{2 N_{r}} \Omega_{i}^{2}, \quad u_{\varphi}=b \sum_{i=1}^{2 N_{r}} l_{i} \mathrm{~s}_{\alpha_{i}} \Omega_{i}^{2}, \\
u_{\theta}=-b \sum_{i=1}^{2 N_{r}} l_{i} \mathrm{c}_{\alpha_{i}} \Omega_{i}^{2}, \quad u_{\psi}=d \sum_{i=1}^{2 N_{r}} S_{p i} \Omega_{i}^{2}
\end{array}
$$

In matrix notation

$$
u=\Theta \Sigma
$$

where $\Sigma \in \mathbb{R}^{2 N_{r}}$ is the vector containing the squared rotor speeds:

$$
\Sigma=\left[\Omega_{1}^{2}, \ldots, \Omega_{2 N_{r}}^{2}\right]^{T}
$$

and $\Theta \in \mathbb{R}^{4 \times 2 N_{r}}$ is the control allocation matrix:

$$
\Theta=\left[\begin{array}{ccccc}
b & \ldots & b & \ldots & b \\
b l_{1} \mathrm{~s}_{\alpha_{1}} & \ldots & b l_{i} \mathrm{~s}_{\alpha_{i}} & \ldots & b l_{2 N_{r}} \mathrm{~s}_{\alpha_{2 N_{r}}} \\
b l_{1} \mathrm{c}_{\alpha_{1}} & \ldots & b l_{i} \mathrm{c}_{\alpha_{i}} & \ldots & b l_{2 N_{r}} \mathrm{c}_{\alpha_{2 N_{r}}} \\
d S_{p 1} & \ldots & d S_{p i} & \ldots & d S_{p 2 N_{r}}
\end{array}\right]
$$


The next step is to deduce the rotational speeds $\Omega_{i}$ from the four desired control inputs that are delivered by the controller. This mapping between the desired inputs $u$ and the speeds of the rotors is denoted usually by a mixer matrix $\Theta$.

The fact that the mixer matrix is preprogrammed on the multirotor and for implementation purposes, the desired input vector $u$ can be considered directly in the simulation model instead of the rotors speeds avoiding the use of the mixer matrix. $u$ will be provided by the controller.

Usually, simplified version of the complete dynamic model is considered as oriented control model for the sake of simpler control laws that could be implemented without difficulty in real time. Moreover, via the simplified model, we show the efficiency of the control strategies that can deal with the unmodeled and neglected dynamics and the disturbances. Hereafter, we simplify the multirotor model, neglecting some effects that have a minor impact in conditions of flying with low speed such as hub forces, blade-flapping moments, the gyroscopic moments, etc. Therefore, we keep just the forces and the moments stemming from the rotors. Moreover, accepting approximation (11), dynamic model (4), in terms of position $\chi$, rotation $\eta$ and input vector $u$, can be simplified as:

$$
\left\{\begin{aligned}
\ddot{x} & =u_{z} \frac{\mathrm{c}_{\psi} \mathrm{s}_{\theta} \mathrm{c}_{\varphi}+\mathrm{s}_{\psi} \mathrm{s}_{\varphi}}{m}+F_{\delta_{x}} \\
\ddot{y} & =u_{z} \frac{\mathrm{s}_{\psi} \mathrm{s}_{\theta} \mathrm{c}_{\varphi}-\mathrm{c}_{\psi} \mathrm{s}_{\varphi}}{m}+F_{\delta_{y}} \\
\ddot{z} & =-g+u_{z} \frac{\mathrm{c}_{\theta} \mathrm{c}_{\varphi}}{m}+F_{\delta_{z}} \\
\ddot{\varphi} & =\frac{\left(I_{y}-I_{z}\right)}{I_{x}} \dot{\theta} \dot{\psi}+\frac{u_{\varphi}}{I_{x}}+F_{\delta_{\varphi}} \\
\ddot{\theta} & =\frac{\left(I_{z}-I_{x}\right)}{I_{y}} \dot{\varphi} \dot{\psi}+\frac{u_{\theta}}{I_{y}}+F_{\delta_{\theta}} \\
\ddot{\psi} & =\frac{\left(I_{x}-I_{y}\right)}{I_{z}} \dot{\varphi}+\frac{u_{\psi}}{I_{z}}+F_{\delta_{\psi}}
\end{aligned}\right.
$$

where $\left.F_{\delta_{i}}\right|_{i=x, y, z, \varphi, \theta, \psi} \in \mathbb{R}$ denotes the neglected dynamics, uncertainties and external disturbances.

\section{E. Control architecture}

Our control is designed to ensure the tracking of the desired trajectory along the three axes $\left(x_{r}, y_{r}, z_{r}\right)$ and the yaw angle $\psi_{r}$ (3D autonomous flight). These reference trajectories are provided on-line by a Trajectory Generator Block (TGB) located in the guidance module. In fact, having two sub-systems (translational and rotational) in cascade with different dynamics is not an easy control problem especially in conditions of under-actuation. Therefore, we consider that $x$ and $y$ are controlled through two virtual inputs $u_{x}$ and $u_{y}$ that drive the system to reach $x_{r}$ and $y_{r}$. The control structure scheme is shown in Figure 6. From model (19), the two virtual inputs are selected to be

$$
\begin{aligned}
& u_{x}=c_{\psi} s_{\theta} c_{\varphi}+s_{\psi} s_{\varphi} \\
& u_{y}=s_{\psi} s_{\theta} c_{\varphi}-c_{\psi} s_{\varphi}
\end{aligned}
$$

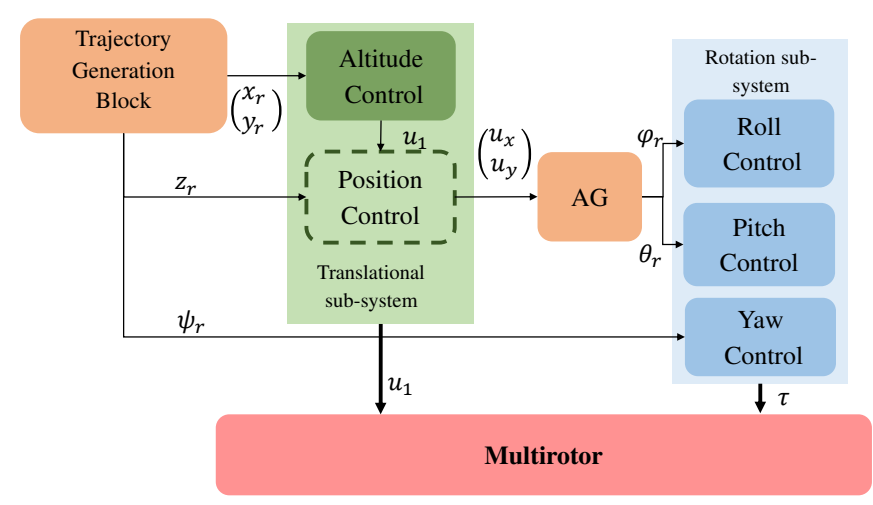

Fig. 6: Control architecture.

From these virtual inputs (20), we compute the desired angles: $\varphi_{r} \neq \pm \frac{\pi}{2}$ and $\theta_{r} \neq \pm \frac{\pi}{2}$. They are considered as inputs for the rotation subsystem and given by

$$
\begin{aligned}
\varphi_{r} & =\arcsin \left(\operatorname{sat}\left(u_{x} \mathrm{~s}_{\psi_{r}}-u_{y} \mathrm{c}_{\psi_{r}}\right)\right) \\
\theta_{r} & =\arcsin \left(\operatorname{sat}\left(\frac{u_{x} \mathrm{c}_{\psi_{r}}+u_{y} \mathrm{~s}_{\psi_{r}}}{\mathrm{c}_{\varphi_{r}}}\right)\right)
\end{aligned}
$$

These reference trajectories $\varphi_{r}$ and $\theta_{r}$ are controlled using $u_{\varphi}$ and $u_{\theta}$ respectively. The altitude is controlled by $u_{z}$ and the yaw angle is controlled by $u_{\psi}$. Saturation function sat(.) is used to avoid the numerical problems, may occur during the implementation of the function $\arcsin ($.$) that has$ a domain $[-1,1]$ and a range $\left[-\frac{\pi}{2},+\frac{\pi}{2}\right]$.

Adopting this control architecture, we are able to imitate a full-actuated system ${ }^{1}$ and to simplify the design of the controller. Therefore, the system (19) can be divided into six independent second order SISO systems with similar structure, in which, the external disturbances, unmodeled dynamics, and coupling terms are merged into a generalized disturbance.

$$
\ddot{q}(t)=\bar{F}_{q}(t)+F_{\delta_{q}}(t)+\beta_{q}(t) u_{i}(t)
$$

with $q=x, y, z, \varphi, \theta, \psi$ where $\bar{F}_{q}(t), F_{\delta_{q}}(t)$ and $\beta_{q}(t)$ can be identified from system (19).

For each SISO system, a robust sliding mode based MFC is developed (SMC-MFC). This controller is discussed in the following section.

\section{Controller DESiGN}

\section{A. Review on Model-Free Control}

According to the literature, the whole dynamics even the external disturbances are approximated by an ultra-local differential relation of order $\nu>0$ valid only on a very short time period $\delta_{t}$ written usually under the following form:

$$
y^{(\nu)}(t)=F(t)+\beta u(t)
$$

where $y$ is the system output, $F$ represents the system dynamic, $u$ is the control input, and $\beta$ is a scale parameter.

\footnotetext{
${ }^{1}$ It is just a mathematical formulation. The multirotor remains underactuated with four inputs and six degrees of freedom.
} 
Usually, order $\nu$ can be 1 or $2 . \beta$ and $\nu$ are chosen by the practitioner.

Many strategies are developed to estimate the unknown dynamic $F(t)$ at instant $t$ such as the sliding mode based observer proposed in [19]. Using directly the experimentally available data, without any modeling step based on the past captured output $y\left(t-\delta_{t}\right)$ and the last applied control input $u\left(t-\delta_{t}\right), F(t) \approx \widehat{F}(t)$ can be estimated as

$$
\begin{aligned}
\widehat{F}(t) & =F\left(t-\delta_{t}\right) \\
& =y^{(\nu)}\left(t-\delta_{t}\right)-\beta u\left(t-\delta_{t}\right)
\end{aligned}
$$

where $\delta_{t}$ is sufficiently small.

Several strategies can be used to differentiate $y$ (see [20]). The next applied control input $u(t)$ at instant $t$ is computed using a simple feedback principle on anticipated model (23) employing the estimated dynamics $\widehat{F}(t)$.

$$
u(t)=-\frac{\widehat{F}(t)-y_{r}^{(\nu)}(t)-v(t)}{\beta}
$$

where $y_{r}(t)$ is the output reference trajectory and $v(t)$ denotes the auxiliary input that ensures the stability of the controlled system.

In the literature, the use of MFC has been linked to the linear PID or PD controllers under the name intelligent-PID ( $i \mathrm{PID})$ and $i \mathrm{PD}$ for second or first order systems respectively. For more details, the reader may refer to [21].

For the rest of paper, we are interested by the second order, $\nu=2$, systems. Therefore, the auxiliary input is written as

$$
v(t)=K_{P} e(t)+K_{I} \int_{0}^{t} e(\tau) d \tau+K_{D} \dot{e}(t)
$$

where $e(t)=y_{r}(t)-y(t)$ is the tracking error. $K_{P}, K_{I}$ and $K_{D}$ denote the usual proportional integral derivative tuning gains. They are positive constants.

The intelligent-PID is written then as

$$
u(t)=\frac{\widehat{F}(t) \quad \ddot{y}_{r}(t) \quad K_{P} e(t) \quad K_{I} \int_{0}^{t} e(\tau) d \tau \quad K_{D} \dot{e}(t)}{\beta}
$$

From equation (27), we quickly recognize that the control of a system, based on the model-free, looks like the solution minimizing the tracking errors at each sample through the estimation of some quantities. It is related to the linearization by feedback principle and the calculation of the PID controllers.

\section{B. Analysis and discussion}

To analyze the $i$ PID performances, controller (27) is applied to system (23) for $\nu=2$. The closed-loop system is written as

$$
\ddot{e}(t)+K_{D} \dot{e}(t)+K_{P} e(t)+K_{I} \int_{0}^{t} e(\tau) d \tau=\tilde{F}(t)
$$

where $\tilde{F}(t)=\widehat{F}(t)-F(t)$ is the estimation error between the real dynamics of the system and the estimated one. The gains $K_{P}, K_{D}$ and $K_{I}$ are selected according to the Hurwitz criterion $^{2}$ to ensure the stability of the system, $\tilde{F}(t)$ is mostly assumed bounded and should be small.

Obviously, from equation (28), we recognize that the performance of MFC depends on the accuracy of the timedelay estimation where the smaller $\delta_{t}$, the better is the estimation $\widehat{F}(t)$.

Because of the time delay $\delta_{t}$, the tracking error $e(t)$ will never converge to zero but will remain in the neighborhood of the zero. The size of this neighborhood depends obviously on the boundedness of $\tilde{F}(t)$. In the following, we will combine the above method with the sliding mode technique, which makes possible to eliminate bounded disturbances, increase the robustness level and improve the performance of the control.

\section{Revisited model free control}

In one hand, implementing a model based nonlinear control technique requires a deep study of the system nonlinearities, where extracting the complete model is almost an impossible task to be achieved. Usually, the provided models are quite simplified with neglected and unmodeled dynamics. In the other hand, assuming no available model is not totally a correct assumption due to the fact that most of systems, at least, may be approximated by mathematical models even with poorly known dynamics where the available information about the system will bring a notable benefit in the control design. As results, we prefer to employ the model-free principle to deal just with the unknown parts of the controlled system (unmodeled dynamics and disturbances) and boosting the capabilities of a nominal model-based control scheme that deals with the known nonlinear modeled dynamics. Therefore, the term $F(t)$ in (23) can be divided into two main components and can be written under a new form:

$$
\ddot{y}(t)=\bar{F}(t)+F_{\delta}(t)+\beta(t) u(t)
$$

where $\bar{F} \in \mathbb{R}$ denotes the known nominal modeled dynamics, $\beta(t) \in \mathbb{R}$ denotes the inputs function and $F_{\delta} \in \mathbb{R}$ represents an additive term gathering all the uncertainties, the neglected and the unmodeled dynamics and the external disturbances.

To determine $F_{\delta}$, we proceed by the MFC principle. Similarly, using the experimentally available data, based on the past captured output $y\left(t-\delta_{t}\right)$ and the last applied control input $u\left(t-\delta_{t}\right)$ in the previous time interval, $F_{\delta}$ can be estimated at each instant $t$ as

$$
\begin{aligned}
\widehat{F}_{\delta}(t) & =F_{\delta}\left(t-\delta_{t}\right) \\
& =\ddot{y}\left(t-\delta_{t}\right)-\beta u\left(t-\delta_{t}\right)-\bar{F}(t)
\end{aligned}
$$

where $\delta_{t}$ is sufficiently small. This estimation is valid for a short period $\delta_{t}$ only and it should be continuously updated at every iteration of the closed-loop controller. This updated term $\widehat{F}_{\delta}$ captures the unknown dynamics of the system as well as the disturbances during each period $\delta_{t}$.

\footnotetext{
${ }^{2}$ i.e. the roots of the polynomial $P(s)=s^{3}+K_{D} s^{2}+K_{P} s+K_{I}$ are all on the open left-half side of the complex plane.
} 
From (29), the control input to be applied is derived from a simple dynamic inversion and can be split into two parts:

$$
u(t)=\frac{1}{\beta(t)} \bar{u}(t)+\frac{\beta_{\delta}}{\beta(t)} u_{\delta}(t)
$$

where $\beta_{\delta}>0$ is fixed by the practitioner and $\bar{u}(t)=$ $-\bar{F}(t)$ plays the role of compensator of the known part. $u_{\delta}(t)$ is considered as an auxiliary input that deals with the disturbances and uncertainties and ensures the asymptotic convergence of the tracking errors of the closed-loop into the origin.

This strategy is not pure data-driven, i.e. the differential equations associated to the mathematical physical laws are used to design the controller and the input and output data are used for accommodate the unknown parts.

Injecting input (31) in (29), leads to a new and fully unknown model eliminating the known part.

$$
\ddot{y}(t)=F_{\delta}(t)+\beta_{\delta} u_{\delta}(t)
$$

Clearly, equation (32) is similar to ultra-local model discussed in Sections IV-A-IV-B. Therefore, motivated by equation (27), the control law for system (32) can be written as

$$
u_{\delta}(t)=\frac{\widehat{F}_{\delta}(t) \quad \ddot{y}_{r}(t) \quad K_{P} e(t) \quad K_{I} \int_{0}^{t} e(\tau) d \tau \quad K_{D} \dot{e}(t)}{\beta_{\delta}}
$$

However, this formulation is highly related to precision of the estimated unknown term where a steady-state error persists (see equation (28)). Therefore, an extra effort $v(t) \in$ $\mathbb{R}$ is required where (33) becomes

$$
\begin{aligned}
u_{\delta}(t)= & -\frac{\beta_{\delta} v(t)+\widehat{F}_{\delta}(t)-\ddot{y}_{r}(t)}{\beta_{\delta}} \\
& +\frac{K_{P} e(t)+K_{I} \int_{0}^{t} e(\tau) d \tau+K_{D} \dot{e}(t)}{\beta_{\delta}}
\end{aligned}
$$

Substituting (34) in (32), we get

$\ddot{e}(t)+K_{P} e(t)+K_{I} \int_{0}^{t} e(\tau) d \tau+K_{D} \dot{e}(t)=\tilde{F}_{\delta}(t)+\beta_{\delta} v(t)$

We stress that $\tilde{F}_{\delta}(t)$ is totally unknown but assumed to be bounded $\left(\left|\tilde{F}_{\delta}(t)\right| \leq \Delta_{\max }\right)$.

The additional input $v(t)$ is designed to compensate the disturbance. Thus, we investigate the sliding mode framework where the sliding surface is defined in state space as:

$$
\mathcal{S}(t)=\dot{e}(t)+\lambda e(t)
$$

where $\lambda$ is a positive gain to adjust the rate of convergence.

The first time derivative of $\mathcal{S}(t)$ is

$$
\dot{\mathcal{S}}(t)=\ddot{e}(t)+\lambda \dot{e}(t)
$$

Substituting $\ddot{e}$ from (35) to equation (37), we get

$$
\dot{\mathcal{S}}(t)=\tilde{F}_{\delta}(t)+\beta_{\delta} v(t)+\lambda \dot{e}(t) \quad K_{P} e(t) \quad K_{I} \int_{0}^{t} e(\tau) d \tau \quad K_{D} \dot{e}(t)
$$

Usually, the sliding mode controller contains an equivalent term $v_{e q}$ occurs on the sliding surface $\mathcal{S}=0$ and a switching term $v_{s w}$, which drives the system states toward the sliding mode. A possible choice of the sliding-mode controller is the following

$$
\begin{aligned}
v & =v_{e q}+v_{s w} \\
& =v_{e q}-\gamma_{1} \mathcal{S}-\gamma_{2} \operatorname{sgn}(\mathcal{S})
\end{aligned}
$$

By making $\mathcal{S}(t)=0$, we determine the equivalent control term by injecting control law (39) into (38). Doing some computations, we obtain the following controller.

$$
\begin{aligned}
v(t)= & \frac{1}{\beta_{\delta}}\left(-\lambda \dot{e}(t)+K_{P} e(t)+K_{I} \int_{0}^{t} e(\tau) d \tau+K_{D} \dot{e}(t)\right. \\
& \left.-\Delta_{\max }\right)-\gamma_{1} \mathcal{S}-\gamma_{2} \operatorname{sgn}(\mathcal{S})
\end{aligned}
$$

The overall control to be applied is then

$$
\begin{aligned}
u(t)= & \frac{1}{\beta(t)}\left(-\bar{F}(t)+\lambda \dot{e}(t)+\Delta_{\max }+\beta_{\delta} \gamma_{1} \mathcal{S}+\beta_{\delta} \gamma_{2} \operatorname{sgn}(\mathcal{S})\right. \\
& \left.-\widehat{F}_{\delta}(t)+\ddot{y}_{r}(t)\right)
\end{aligned}
$$

This controller has several benefits such as the simplicity of its structure. Moreover, it presents a very good level of robustness with self-adaption in the case of disturbances. This new formulation that combines the MFC and the sliding mode and takes into consideration the system information has potential advantages in control performance compared to the classical controllers.

\section{Stability analysis}

The stability of controller system (41) is summarized by the flowing theorem.

Theorem: Closed-loop of disturbed system (29) using controller (41) is asymptotically stable provided that $\beta_{\delta} \gamma_{2}>2 \Delta_{\max }$ where $\beta_{\delta}$ and $\gamma_{2}$ are positive parameters.

Proof: Substituting controller (41) into system dynamics (29) yields

$$
\ddot{e}(t)=\tilde{F}_{\delta}(t)-\Delta_{\max }-\lambda \dot{e}(t)-\beta_{\delta} \gamma_{1} \mathcal{S}-\beta_{\delta} \gamma_{2} \operatorname{sgn}(\mathcal{S})
$$

Let $V(t)$ a Lyapunov candidate function. It equals

$$
V(t)=\frac{1}{2} \mathcal{S}(t)^{2}
$$

The first time derivative of $V$ yields

$$
\dot{V}(t)=\mathcal{S}(t) \dot{\mathcal{S}}(t)
$$

Using equation (38) and (42), yields

$$
\begin{aligned}
\dot{V}(t) & =\mathcal{S}(t) \dot{\mathcal{S}}(t) \\
& =\mathcal{S}(t)(\ddot{e}(t)+\lambda \dot{e}(t)) \\
& =\mathcal{S}(t)\left(\tilde{F}_{\delta}(t)-\Delta_{\max }-\beta_{\delta} \gamma_{1} \mathcal{S}-\beta_{\delta} \gamma_{2} \operatorname{sgn}(\mathcal{S})\right)
\end{aligned}
$$


$\dot{V}(t)$ in equation (45) can be rewritten under the following form

$$
\dot{V}(t)=-\beta_{\delta} \gamma_{1} \mathcal{S}^{2}-\beta_{\delta} \gamma_{2}|\mathcal{S}|-\mathcal{S}(t)\left(\Delta_{\max }-\tilde{F}_{\delta}(t)\right)
$$

Therefore, according to the boundedness of $\tilde{F}_{\delta}$, it is ensured that $\dot{V}<0$ if one has $\beta_{\delta} \gamma_{2}>2 \Delta_{\max }$. This implies that the right-side term is negative which achieves the asymptotic stability condition.

\section{RESULTS AND DISCUSSION}

In this section, we investigate the efficiency of the proposed controller through and application for an X-shaped quadrotor where its parameters are depicted in Table I.

TABLE I: Quadrotor parameters [22].

\begin{tabular}{|c|c|c|c|}
$m(\mathrm{~kg})$ & 0.429 & $I_{y}\left(\mathrm{~kg} \cdot \mathrm{m}^{2}\right)$ & 0.0029 \\
\hline$I_{x}\left(\mathrm{~kg} \cdot \mathrm{m}^{2}\right)$ & 0.0022 & $I_{z}\left(\mathrm{~kg} \cdot \mathrm{m}^{2}\right)$ & 0.0048
\end{tabular}

The controller is designed considering the simplified model (19) and applied to complete system (4). Its effectiveness is investigated in the presence, at the same time, of different disturbances namely: model parameters uncertainties supposing the inertia matrix elements are underrated 50\% of the nominal values, sensors noise by adding random noise on the states of the quadrotor and gust of wind. Here, we accept that the wind causes the same acceleration intensity $a_{x}, a_{y}$ and $a_{z}$ on all $X_{E}, Y_{E}, Z_{E}$-axes expressed in the Earth-fixed frame $R_{E}$. These accelerations are considered as disturbances added to the equations related to the forces in the quadrotor model. Therefore, the disturbed model is expressed as follows

$$
\left\{\begin{array}{l}
\ddot{\tilde{x}}=\ddot{x}+a_{x}(t) \\
\ddot{\tilde{y}}=\ddot{y}+a_{y}(t) \\
\ddot{\tilde{z}}=\ddot{z}+a_{z}(t)
\end{array}\right.
$$

The profile of this accelerations is depicted in Figure 7.

After the take-off, the quadrotor tracks a square reference trajectory of width $L_{r}=2 m$ (see Figure 8). When it arrives at each corner, the quadrotor hovers for five seconds then it flies to the next corner where the flight duration between two corners is fixed at $T=5$ seconds. Therefore, this trajectory may be described as

$$
\sigma(t)=\left\{\begin{array}{lll}
0 & \text { when } 0 \leq t \leq t_{1} \\
L_{r} \frac{\left(t-t_{1}\right)^{5}}{\left(t-t_{1}\right)^{5}+\left(T-t+t_{1}\right)} & \text { when } t_{1}<t \leq t_{2} \\
L_{r} & \text { when } t_{2}<t \leq t_{3} \\
L_{r}-L_{r} \frac{\left(t-t_{3}\right)^{5}}{\left(t-t_{3}\right)^{5}+\left(T-t+t_{3}\right)} & \text { when } t_{3}<t \leq t_{4} \\
0 & \text { when } t_{4}<t \leq t_{f}
\end{array}\right.
$$

with $T=5$ seconds, $t_{f}=80$ seconds and $L_{r}=2$ meters. $x_{r}=\sigma(t)$ with $t_{1}=5, t_{2}=t_{1}+T, t_{3}=25, t_{4}=t_{3}+T$ $y_{r}=\sigma(t)$ with $t_{1}=10, t_{2}=t_{1}+T, t_{3}=30, t_{4}=t_{3}+T$ $z_{r}=\sigma(t)$ with $t_{1}=0, t_{2}=t_{1}+T, t_{3}=35, t_{4}=t_{3}+T$ $\psi_{r}=0$

The control parameters used for the sumulations are given by $K_{P}=19.414, K_{I}=8.632, K_{D}=0.691, \beta_{\delta}=2.1$, $\gamma_{1}=5, \gamma_{2}=0.1$ and $\lambda=5.2$.

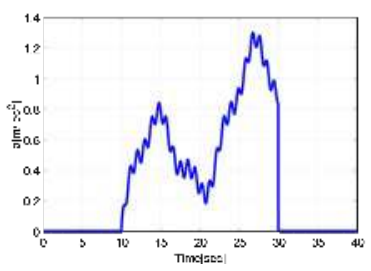

Fig. 7: Wind disturbance profile.

TABLE II: Metric comparison

\begin{tabular}{|c|l|l|}
\hline & MFC & SMC-MFC \\
\hline ISE & 0.0037 & 0.0018 \\
\hline ISCI & $1.4033 \mathrm{e}+03$ & $1.4204 \mathrm{e}+03$ \\
\hline
\end{tabular}

We have developed a Matlab simulation environment to evaluate the effectiveness of the proposed control strategy applied to the quadrotor. The results are depicted in Figures 9-10. In each figure, we plot separately, the tracking errors of the translations along $X_{E}, Y_{E}, Z_{E}$-axes, the attitude angles $\varphi, \theta$ and $\psi$ and the control inputs (global thrust and torques).

From the curves displayed in Figures 9-10, the quadrotor follows its reference trajectory in a good manner and satisfactory accuracy. Clearly, the SMC-MFC exhibits a damped response for which the overshoot is the smallest (see Figure 9). Moreover, the control, using SMC-MFC, is more accurate compared to the classic MFC. However, it consumes more energy. We stress that SMC-MFC is sensitive to noise as shown in Figure 10 due to the discontinuous functions used by this controller namely $s g n$ function.

The previous observations are confirmed by some statistical analysis metrics, which are the Integral Square Error (ISE) for the accuracy and the Integral square input control (ISCI) for the consumed energy. The obtained values are depicted in Table II.

\section{CONCLUSION}

Within this paper, a robust control strategy is developed and applied to generic model of multirotors. The strategy is an alternative to the popular model-free control as a revisited form. The basic MFC was investigated to deal with the uncertain part of the system. Moreover, the proposed control approach used the sliding mode controller in order to raise the performance of the control even in presence of disturbances. Satisfactory results were obtained in numerical simulations that will serve as a first step to pursue by experimental tests in the near future.

\section{REFERENCES}

[1] Y. Bouzid, Y. Bestaoui, and H. Siguerdidjane, "Quadrotor-UAV optimal coverage path planning in cluttered environment with a limited onboard energy," in 2017 IEEE/RSJ International Conference on Intelligent Robots and Systems (IROS), Sept. 2017, pp. 979-984.

[2] K. Nonami, F. Kendoul, S. Suzuki, P. W. Wang, and D. Nakazawa, Autonomous Flying Robots: Unmanned Aerial Vehicles and Micro Aerial Vehicles, 1st ed. Tokyo ; New York: Springer Verlag, Japan, Mar. 2010. 

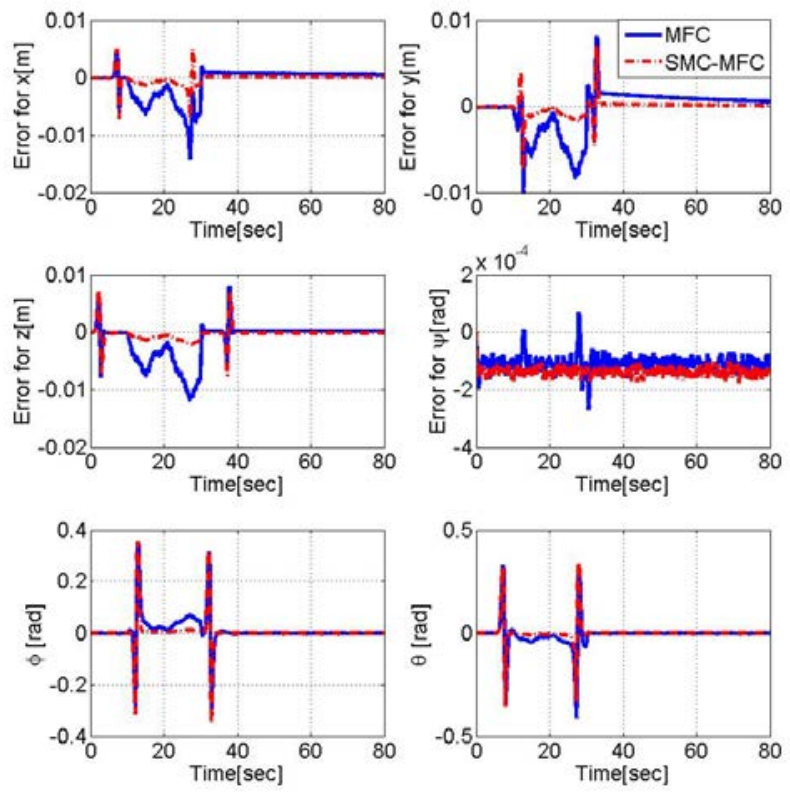

Fig. 9: Outputs of the quadrotor.
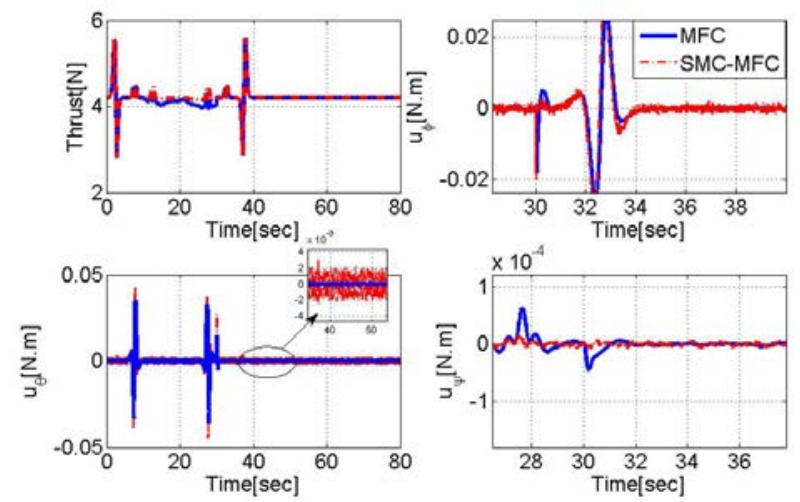

Fig. 10: Control inputs.

[3] Y. Bouzid, H. Siguerdidjane, and Y. Bestaoui, "Nonlinear internal model control applied to VTOL multi-rotors UAV," Mechatronics, vol. 47, pp. 49-66, Nov. 2017. [Online]. Available: http://www.sciencedirect.com/science/article/pii/S0957415817301046

[4] Y. Bouzid, H. Siguerdidjane, Y. Bestaoui, and M. Zareb, "Energy Based 3D Autopilot for VTOL UAV Under Guidance \&; Navigation Constraints," J Intell Robot Syst, pp. 1-22, Nov. 2016.

[5] Y. Bouzid, H. Siguerdidjane, and Y. Bestaoui, "Hierarchical Autopilot Design based on Immersion \& Invariance and Nonlinear Internal Model Tracking Controllers for Autonomous system," IFACPapersOnLine, vol. 49, no. 5, pp. 103-108, 2016.

[6] J. Alvarenga, N. I. Vitzilaios, K. P. Valavanis, and M. J. Rutherford, "Survey of Unmanned Helicopter Model-Based Navigation and Control Techniques," Journal of Intelligent \& Robotic Systems, vol. 80, no. 1, pp. 87-138, Oct. 2015. [Online]. Available: https://link.springer.com/article/10.1007/s10846-014-0143-5

[7] D. Cabecinhas, R. Cunha, and C. Silvestre, "A nonlinear quadrotor trajectory tracking controller with disturbance rejection," Control Engineering Practice, vol. 26, pp. 1-10, May 2014. [Online]. Available: http://www.sciencedirect.com/science/article/pii/S0967066114000343

[8] X. Gong, Y. Tian, Y. Bai, and C. Zhao, "Trajectory tacking control of a quad-rotor based on active disturbance rejection control," in 2012 IEEE International Conference on Automation and Logistics, Aug. 2012, pp. 254-259.
[9] M. O. Efe, "Robust low altitude behavior control of a quadrotor rotorcraft through sliding modes," in 2007 Mediterranean Conference on Control Automation, June 2007, pp. 1-6.

[10] Y. Bouzid, H. Siguerdidjane, and Y. Bestaoui, "3d trajectory tracking control of quadrotor UAV with on-line disturbance compensation," in 2017 IEEE Conference on Control Technology and Applications (CCTA), Aug. 2017, pp. 2082-2087.

[11] W. F. Hu, C. H. Chen, Y. F. Peng, and C. M. Lin, "Intelligent Robust Control for Three-Link Robot Manipulator via Sliding Mode Technology," in 2007 IEEE 22nd International Symposium on Intelligent Control, Oct. 2007, pp. 499-504.

[12] L. x. Ma, D. n. Shi, M. x. Chen, and X. q. Wang, "Application of intelligent PID control for robot," in 2008 IEEE Conference on Cybernetics and Intelligent Systems, Sept. 2008, pp. 455-458.

[13] Y. Bouzid, H. Siguerdidjane, and Y. Bestaoui, "Flight control boosters for three-dimensional trajectory tracking of quadrotor: Theory and experiment," Proceedings of the Institution of Mechanical Engineers, Part I: Journal of Systems and Control Engineering, p. 0959651818757159, Mar. 2018. [Online]. Available: https://doi.org/10.1177/0959651818757159

[14] E. Altug, J. P. Ostrowski, and R. Mahony, "Control of a quadrotor helicopter using visual feedback," in Proceedings 2002 IEEE International Conference on Robotics and Automation (Cat. No.02CH37292), vol. 1, 2002, pp. 72-77 vol.1.

[15] J. Wang, "Quadrotor analysis and model free control with comparisons," phdthesis, Université Paris Sud - Paris XI, Nov. 2013. [Online]. Available: https://tel.archives-ouvertes.fr/tel00952401/document

[16] "Wiley: Aircraft Control and Simulation: Dynamics, Controls Design, and Autonomous Systems, 3rd Edition - Brian L. Stevens, Frank L. Lewis, Eric N. Johnson."

[17] M. Bangura and R. Mahony, "Nonlinear Dynamic Modeling for High Performance Control of a Quadrotor," in Proceedings Australasian Conference on Robotics and Automation 2012. Australian Robotics and Automation Association, 2012. [Online]. Available: https://openresearch-repository.anu.edu.au/handle/1885/65230

[18] D. Mellinger and V. Kumar, "Minimum snap trajectory generation and control for quadrotors," in 2011 IEEE International Conference on Robotics and Automation, May 2011, pp. 2520-2525.

[19] H. P. Wang, G. I. Y. Mustafa, and Y. Tian, "Modelfree fractional-order sliding mode control for an active vehicle suspension system," Advances in Engineering Software, vol. 115, pp. 452-461, Jan. 2018. [Online]. Available: http://www.sciencedirect.com/science/article/pii/S0965997817308529

[20] Y. A. Younes, A. Drak, H. Noura, A. Rabhi, and A. E. Hajjaji, "Modelfree control of a quadrotor vehicle," in 2014 International Conference on Unmanned Aircraft Systems (ICUAS), May 2014, pp. 1126-1131.

[21] J. Wang, M. S. Geamanu, A. Cela, H. Mounier, and S. I. Niculescu, "Event driven model free control of quadrotor," in 2013 IEEE International Conference on Control Applications (CCA), Aug. 2013, pp. $722-727$.

[22] Y. Bouzid, H. Siguerdidjane, and Y. Bestaoui, "Revisited modelfree controller for $3 \mathrm{~d}$ autonomous navigation of quadrotor: Features analysis in harsh flight conditions," in 2017 13th IEEE International Conference on Control Automation (ICCA), July 2017, pp. 915-920. 